\title{
Comparative study of three types of mesenchymal stem cell to differentiate into pancreatic $\beta$-like cells in vitro
}

\author{
YUNFANG MO $^{1}$, ZEJIAN WANG ${ }^{1}$, JIAN GAO ${ }^{1}$, YAN YAN $^{1}$, HUAIJUAN REN ${ }^{1}$, \\ FENGLI ZHANG $^{1}$, NIANMIN QI $^{2}$ and YANTIAN CHEN ${ }^{1}$
}

\author{
${ }^{1}$ Engineering Research Center of Cell and Therapeutic Antibody, Ministry of Education, School of Pharmacy, \\ Shanghai Jiao Tong University, Shanghai 200240; ${ }^{2}$ China Stem Cell Therapy Co., Ltd., Shanghai 201203, P.R. China
}

Received October 14, 2020; Accepted May 11, 2021

DOI: $10.3892 / \mathrm{etm} .2021 .10368$

\begin{abstract}
Type 1 diabetes (TID) is a chronic metabolic disease where the body produces insufficient or no insulin. Stem cells with multi-directional differentiation potential are transplanted and differentiate into $\beta$-like cells in vivo to replace pancreatic $\beta$ cells, which has become a novel treatment strategy. The aim of the present study was to investigate the ability of three types of adult mesenchymal stem cell (MSC) to differentiate into pancreatic $\beta$-like cells in vitro in order to identify suitable sources for the treatment of diabetes. The three MSC types were menstrual blood-derived MSCs (MENSCs), umbilical cord-derived MSCs (UCMSCs) and dental pulp MSCs (DPSCs). The differentiation method used in the present study was divided into three steps and the MSCs were differentiated into pancreatic $\beta$-like cells in vitro. Among these MSCs, MENSCs had a greater ability to differentiate into islet $\beta$-like cells in vitro, while UCMSCs and DPSCs exhibited a similar differentiation potency, which was relatively lower compared with that of MENSCs. The present results indicated that MENSCs may be a suitable cell source for the curative treatment of TID.
\end{abstract}

Correspondence to: Dr Yantian Chen, Engineering Research Center of Cell and Therapeutic Antibody, Ministry of Education, School of Pharmacy, Shanghai Jiao Tong University, 800 Dongchuan Road, Minhang, Shanghai 200240, P.R. China

E-mail: ytchen@sjtu.edu.cn

Abbreviations: MSC, mesenchymal stem cell; MENSCs, menstrual blood-derived MSCs; UCMSCs, umbilical cord MSCs; DPSCs, dental pulp MSCs; FBS, fetal bovine serum; SC- $\beta$ cell, stem-cell-derived $\beta$-cell; DAPI, 4',6-diamidino-2-phenylindole; GLUT-2, glucose transporter 2; NGN-3, neurogenin 3; DE, definitive endoderm

Key words: mesenchymal stem cell, cell differentiation, diabetes mellitus, pancreatic $\beta$-cell, collagen

\section{Introduction}

Diabetes is a chronic disease that occurs when the pancreas is no longer able to provide insulin or when the body cannot make use of the insulin it produces (1). According to data from the International Diabetes Federation, 463 million adults suffered from diabetes in 2019 (https://www.idf.org). Approximately $10 \%$ of them had type 1 diabetes (TID). However, there are still no effective methods for the treatment of the disease. Individuals with TID depend on daily insulin injections to maintain their blood glucose levels. In recent years, the transplantation of stem cells with multi-directional differentiation potential to replace the islet cells has become a novel treatment strategy (2). Clinical trials with mesenchymal stem cell (MSC)-based therapies are currently limited (3). A study using intravenous infusion of bone marrow MSCs (BMMSCs) demonstrated improvement of C-peptide at one year (4), which suggested the potential of MSCs in the treatment of diabetes. The present study first compared the differentiation potential of three types of MSC into islet $\beta$-like cells in vitro.

These three types of MSC were umbilical cord-derived MSCs (UCMSCs), dental pulp MSCs (DPSCs) and menstrual blood-derived MSCs (MENSCs), which were isolated from the Wharton's jelly tissue of the human umbilical cord (5), deciduous teeth (6) and menstrual blood (7), respectively. The three MSC types share advantages in their clinical application, such as convenient harvesting procedures, high proliferation, multi-directional differentiation capacity and low immunogenicity (8). Studies have indicated that UCMSCs may be induced to form cartilage, bone, adipose and nerve cells (9). Numerous studies assessed the differentiation potency of UCMSCs into pancreatic cells in vitro, which proved their differentiation ability (10). In the present study, UCMSCs were used as a judgement standard to compare the differentiation ability of DPSCs and MENSCs. DPSCs are one of the novel types of MSCs that have been proposed for tissue regeneration to repair bone defects (11). DPSCs are obtained from human exfoliated deciduous teeth, having the characteristics of being easy to obtain with little damage caused to donors (12). It has been demonstrated that MENSCs have the ability to differentiate into osteogenic, neurogenic and chondrogenic cell lineages (13), which 
prompts the potential of MENSCs for use in regenerative medicine research. The isolation of MENSCs features reproducibility and no damage to donors, which means the source of MENSCs is rich and they are easy to obtain (7). Previous studies addressed the differentiation ability of the three types of MSC, particularly MENSCs. In addition to comparing the differentiation ability of three types of MSC, it was explored whether MENSCs are able to differentiate into islet cells as a novel clinical application.

As for the methodology, the previous differentiation methods were improved (14), and in combination with the results of the experiments, an optimized differentiation method was established. Collagen coating is used to promote the proliferation and differentiation ability of cells (15), among the different collagens, rat tail collagen is easy to obtain and mostly used $(16,17)$. In this procedure, 12 -well cell culture plates were pre-incubated with rat tail collagen, which improved the differentiation ability in comparison with the previous method without collagen coating.

The biological characteristics of these MSCs, including proliferation, survival and differentiation capacities, were assessed in former studies (18). Other biological characteristics, particularly the evaluation of differentiation ability into pancreatic $\beta$-like cells, were performed in the present study. Although several types of MSC were reported to be able to differentiate into pancreatic $\beta$-like cells in vitro, including BMMSCs, adipose-derived MSCs (ADSCs) and UCMSCs, the curative effect was not ideal in vivo (19-21). The purpose of the present study was to screen novel sources of MSCs for the treatment of diabetes and to provide a new scope for pre-clinical trials in vivo. The present study will aid in future decision-making for choosing suitable seed cells for the treatment of TID in clinical trials.

\section{Materials and methods}

Isolation and culture of MENSCs, UCMSCs and DPSCs. Isolation of MENSCs, UCMSCs and DPSCs was performed at the Cell Culture \& Bioprocess Engineering Laboratory, Shanghai Jiaotong University (Shanghai, China). Relevant materials and samples were provided by Shanghai Kun'ai Biological Technology Co., Ltd., (Shanghai, China). The present study was approved by the Ethics Committee of the School of Pharmacy, Shanghai Jiaotong University (Shanghai, China).

UCMSCs. Umbilical cord tissues were collected from a 35-year-old mother and full-term fetus after caesarean section in Handan Central Hospital (Handan, China) in March 2014. The donor had a physical examination to rule out infectious diseases. The tissues were stored at $4^{\circ} \mathrm{C}$ and transported to Shanghai. The isolation of UCMSCs was performed in the laboratory of Shanghai Jiao Tong University. Collected umbilical cord tissues were washed with PBS several times to remove red blood cells. After Wharton's jelly tissues were removed from umbilical cords, they were chopped into small pieces and placed on $55-\mathrm{mm}^{2}$ plates at $37^{\circ} \mathrm{C}$ for $2 \mathrm{~h}$. The tissues adhered to the plates to avoid suspension of the pieces when medium was added. Then the plates were covered with $\alpha$-MEM medium (Gibco; Thermo Fisher Scientific, Inc.) containing 15\% FBS (HyClone; Cytiva).
MENSCs. Menstrual blood was obtained from a 39-year-old female without abnormal discharge or infection by sterile Diva Cup on the second day of flow in October 2014 at the donor's home (Shanghai, China). The donor was tested for infectious diseases, blood chemistry indicators and ultrasound examinations to ensure health. The isolation of MENSCs was performed in the laboratory of Shanghai Jiao Tong University. The collected menstrual blood $(5 \mathrm{ml})$ was mixed with an equal volume of PBS, $0.2 \mathrm{ml}$ amphotericin B (Invitrogen; Thermo Fisher Scientific, Inc.), $0.2 \mathrm{ml}$ streptomycin (Invitrogen; Thermo Fisher Scientific, Inc.) and $0.1 \mathrm{ml}$ EDTA-Na (Invitrogen; Thermo Fisher Scientific, Inc.) at $4^{\circ} \mathrm{C}$ within $24 \mathrm{~h}$ of collection. Density gradient centrifugation was applied twice at $200 \mathrm{x} \mathrm{g}$ at $4^{\circ} \mathrm{C}$ for $10 \mathrm{~min}$, followed by discarding the supernatant and adding fresh medium containing $15 \% \mathrm{FBS}$ for cell culture.

DPSCs. Dental pulp tissues were obtained from shed deciduous teeth. The tissues were donated from a 15 -year-old male in April 2014 at the donor's home (Shanghai, China). The isolation of DPSCs was operated in the laboratory. The dental pulp tissues were gently separated from the root and the crown. Then, the tissues were minced and dissociated in $0.3 \%$ collagenase type I (cat. no. SCR103; Sigma-Aldrich; Merck KgaA) at $37^{\circ} \mathrm{C}$ for $1 \mathrm{~h}$. Subsequently, dissociation was terminated by adding $\alpha$-MEM with $10 \% \mathrm{FBS}$, followed by centrifugation at $100 \mathrm{x} \mathrm{g}$ at $4^{\circ} \mathrm{C}$ for $5 \mathrm{~min}$. To the sediment, $\alpha$-MEM containing $15 \%$ FBS was added to re-suspend the cells and single cell suspensions were cultured as primary MSCs in 6-well plates at a concentration of $1 \times 10^{5}$ cells/well.

The suspension density of isolated MENSCs, UCMSCs and DPSCs primary culture cells was adjusted to $2 \times 10^{6}$ cells $/ \mathrm{ml}$. These cells were cultured in 6-well cell culture plates. Cells that failed to stick to the walls were discarded after $48 \mathrm{~h}$. The medium was changed every two days. The morphology of growing cells was observed under an inverted phase contrast microscope (Olympus Corporation).

Cell surface markers on MENSCs, UCMSCs and DPSCs. MENSCs, UCMSCs and DPSCs at passage 4 (P4) were trypsinized with $0.25 \%$ TrypLE (Invitrogen; Thermo Fisher Scientific, Inc.), washed twice with PBS (pH 7.4) and suspended in PBS at a concentration of $5 \times 10^{6}$ cells $/ \mathrm{ml}$. The sample was incubated with fluorescein isothiocyanate-conjugated monoclonal antibody CD14 (cat. no. 11-0149-42), CD45 (cat. no. 11-9459-42), isotype control (cat. no. 11-4714-41) (all 1:200; all from eBioscience; Thermo Fisher Scientific, Inc.), or incubated with phycoerythrin-conjugated monoclonal antibody CD29 (cat. no. 555443), CD34 (cat. no. 550761), CD44 (cat. no. 550989) (all 1:50; all from BD Biosciences), CD90 (cat. no. 555596), isotype control (cat. no. 550617) (both 1:100; both from BD Biosciences) for $30 \mathrm{~min}$ at $4^{\circ} \mathrm{C}$ in the dark according to the manufacturer's recommendation. Finally, cells were washed twice with PBS and then analyzed with a standard FACSAria flow cytometer (BD Biosciences) and results were evaluated with CellQuest Pro software v1.0.2 (BD Biosciences).

Growth kinetics of MENSCs, UCMSCs and DPSCs. MENSCs, UCMSCs and DPSCs with $90 \%$ confluency were dissociated with TrypLE. Based on the cell count, $10^{4}$ cells/well were 
seeded in 24-well cell culture plates (Corning Inc.) and cultured at $37^{\circ} \mathrm{C}$ with $5 \% \mathrm{CO}_{2}(\mathrm{v} / \mathrm{v})$ and saturated humidity. After $24 \mathrm{~h}$ of incubation, each of the three cell types were dissociated in the three wells, stained with the same amount of trypan blue (Gibco; Thermo Fisher Scientific, Inc.) and the cell number was then counted with an automatic cell counter (Countstar). These procedures were repeated for the three wells every two days. The medium was changed every two days. Finally, to plot the growth curves for MENSCs, UCMSCs and DPSCs, the culture time was displayed on the abscissa, while the mean number of cells was presented on the ordinate (18).

The population doubling time (PDT) was calculated for these cells according to the following equation:

$$
\mathrm{PDT}=\mathrm{t} \times \frac{\lg 2}{\lg \left(\mathrm{N}_{\mathrm{t}} / \mathrm{N}_{0}\right)}
$$

where $\mathrm{t}$ is the culture time, $\lg$ is $\log _{10}, \mathrm{~N}_{\mathrm{t}}$ the harvested cell number and $\mathrm{N}_{0}$ the cell number at the beginning.

The maximum specific cell growth rate $\left(\mu_{\mathrm{m}}\right)$ was calculated using the PDT value as follows:

$$
\mu_{\mathrm{m}}=\frac{\ln 2}{\mathrm{PDT}}
$$

where $\ln$ is $\log _{\mathrm{e}}$.

Multi-differentiation ability of MENSCs, UCMSCs and DPSCs. For adipogenic differentiation, MENSCs, UCMSCs and DPSCs were cultured in Dulbecco's modified Eagle's medium (DMEM; Gibco; Thermo Fisher Scientific, Inc.) supplemented with high glucose, $10 \%$ fetal bovine serum (FBS; HyClone; Cytiva), $1 \mathrm{mM}$ dexamethasone, $0.5 \mathrm{mM}$ methyl-isobutyl-xanthine, $10 \mathrm{mg} / \mathrm{ml}$ insulin and $100 \mathrm{mM}$ indomethacin (all from Sigma-Aldrich; Merck KGaA) for 4 weeks. The control groups were cultured in DMEM supplemented with high glucose and 10\% FBS. At the end of the incubation, adipogenic differentiation was assayed with $0.3 \%$ Oil red O (Sigma-Aldrich; Merck KGaA) staining for lipid droplets for $1 \mathrm{~h}$ at $37^{\circ} \mathrm{C}$. After observation with an inverted phase-contrast microscope, the stained cells were eluted with $500 \mu \mathrm{l}$ isopropyl alcohol per well for $5 \mathrm{~min}$ at room temperature and then quantified by determining the absorbance at $510 \mathrm{~nm}$ using a microplate reader (Thermo Fisher Scientific, Inc.).

For osteogenic differentiation, MENSCs, UCMSCs and DPSCs were cultured in DMEM supplemented with high glucose, $10 \%$ FBS, $0.1 \mathrm{mM}$ dexamethasone, $10 \mathrm{mM}$ $\beta$-glycerolphosphate and $50 \mathrm{mM}$ ascorbic acid (all from Sigma-Aldrich; Merck KGaA) for 3 weeks. The control groups were cultured in high-glucose DMEM just containing $10 \%$ FBS. At the end of the incubation, osteogenic differentiation was assayed with $0.1 \%$ Alizarin red S (Sigma-Aldrich; Merck $\mathrm{KGaA}$ ) staining for calcium deposition for $1 \mathrm{~h}$ at $37^{\circ} \mathrm{C}$. After observation with an inverted phase-contrast microscope, the stained cells were eluted with $500 \mu 110 \%$ cetylpyridinium chloride per well (Sigma-Aldrich; Merck KGaA) for $10 \mathrm{~min}$ at room temperature and then quantified by measuring the absorbance at $562 \mathrm{~nm}$ using a microplate reader.

Production of pancreatic $\beta$-like cells from MENSCs, UCMSCs and DPSCs using three-step method. Study of the role of inductive substances $(16,22)$, analysis of previous studies reporting differentiation methods from mesenchymal into pancreatic cells and combination with the preliminary experimental data resulted in the design of a differentiation method $(14,23)$, the three-step induction system, to differentiate MENSCs, UCMSCs and DPSCs into islet cells in vitro.

MENSCs, UCMSCs and DPSCs at P3 that grew to 70\% confluency were dissociated, the concentration was adjusted to $10^{5}$ cells $/ \mathrm{ml}$ and cells were seeded in 12-well cell culture plates. The plates were pre-coated with type 1 rat-tail collagen (cat. no. C7661; Sigma-Aldrich; Merck KGaA). The induction procedure was then followed:

Step 1. Following incubation overnight, the medium was changed to high-glucose DMEM containing 10\% FBS and $10^{-6} \mathrm{~mol} / 1$ retinoic acid (Sigma-Aldrich; Merck KGaA) to induce differentiation for $48 \mathrm{~h}$. The medium was then changed to high-glucose DMEM only with $10 \%$ FBS, followed by culture for $24 \mathrm{~h}$.

Step 2. Acetic acid $(0.1 \mathrm{M})$ was used to dissolve type 1 rat tail collagen to a $5 \mathrm{mg} / \mathrm{ml}$ solution. A total of $200 \mu \mathrm{l}$ rat tail collagen solution, was mixed with $798 \mu$ l DMEM medium and $12 \mu \mathrm{l} 0.1 \mathrm{M} \mathrm{NaOH}$. A total of $30 \mu \mathrm{l}$ of this solution was added to each well of 12 -well plates, and placed at $37^{\circ} \mathrm{C}$ for $20 \mathrm{~min}$. The cells were then dissociated with TrypLE and the concentration of the cell suspension was adjusted to $10^{5}$ cells $/ \mathrm{ml}$. The cells were seeded in new 12-well cell culture plates pre-incubated with type 1 rat-tail collagen. MENSCs, UCMSCs and DPSCs were cultured in the induction medium consisting of low-glucose DMEM, 10\% FBS, $10 \mathrm{mmol} / 1$ nicotinamide, $20 \mathrm{ng} / \mathrm{ml}$ epidermal growth factor and $50 \mathrm{ng} / \mathrm{ml}$ basic fibroblast growth factor (all from Sigma-Aldrich; Merck $\mathrm{KGaA}$ ) for 7 days.

Step 3. The medium of the second step was removed and changed to low-glucose DMEM containing exendin 4 (Sigma-Aldrich; Merck KGaA) and 10\% FBS to continue induction for another 7 days.

After differentiation, MSCs were stained with dithizone (DTZ; Sigma-Aldrich; Merck KGaA). DTZ is a zinc-chelating agent known to stain pancreatic $\beta$-cells due to their high zinc content (24). Images were captured with an inverted phase-contrast microscope (Olympus Corporation) and analyzed with ImageJ software v2.0 (National Institutes of Health) imitating the way of immunohistochemistry to obtain quantitative statistics (25). Furthermore, the staining intensity of these images was graded from 1-5 (the weakest stained as 1 and the strongest stained as 5), determined in a double-blinded way by three independent investigators, followed by statistical evaluation (26).

Immunofluorescence staining. Following differentiation, the MSCs were fixed with $4 \%$ paraformaldehyde for $20 \mathrm{~min}$, permeabilized with PBS containing $0.3 \%$ Triton-X and then blocked with PBS containing 5\% goat serum (cat. no. G9023; Sigma-Aldrich; Merck KgaA) for $45 \mathrm{~min}$ at room temperature. Cells were incubated with rabbit monoclonal anti-insulin primary antibody (1:200; cat. no. ab181547; Abcam) at $4^{\circ} \mathrm{C}$ overnight. The three types of cells were 
Table I. Primer sequences designed forquantitative PCR detection of genes to determine the differentiation ability from stem cells into pancreatic $\beta$-like cells.

\begin{tabular}{lll}
\hline Gene name & \multicolumn{1}{c}{ Forward primer (5'-3') } & \multicolumn{1}{c}{ Reverse primer (5'-3') } \\
\hline GAPDH & ATGGGGAAGGTGAAGGTCG & GGGGTCATTGATGGCAACAATA \\
INSULIN & AGCATCTGCTCCCTCTACCA & TGCTGGTTCAAGGGCTTTAT \\
GLUT-2 & CAGCTGTCTTGTGCTCTGCTTGT & GCCGTCATGCTCACATAACTCA \\
NGN-3 & AAGAGCGAGTTGGCACTGAGC & CGTACAAGCTGTGGTCCGC \\
\hline
\end{tabular}

GLUT-2, glucose transporter 2; NGN-3, neurogenin 3.

Table II. Cell populationdoubling time and the largest growth rate of MENSCs, UCMSCs and DPSCs.

\begin{tabular}{lcc}
\hline Cell type & $\begin{array}{c}\text { Population } \\
\text { doubling time (h) }\end{array}$ & $\begin{array}{c}\text { Maximum specific cell } \\
\text { growth rate (s-1) }\end{array}$ \\
\hline MENSC-P4 & $65.40 \pm 12.96$ & 0.0106 \\
UCMSC-P6 & $45.17 \pm 4.22$ & 0.0156 \\
DPSC-P5 & $53.81 \pm 2.64$ & 0.0130 \\
\hline
\end{tabular}

MENSCs, menstrual blood-derived MSCs; UCMSCs, umbilical cord-derived MSCs; DPSCs, dental pulp MSCs; MSC, mesenchymal stem cell; P4, passage 4 .

then washed with PBS and stained with the tetramethylrhodamine (TRITC)-conjugated goat anti-rabbit secondary antibody (1:300; cat. no. A16101; Invitrogen; Thermo Fisher Scientific, Inc.) at room temperature for $1 \mathrm{~h}$ in the dark. Cell nuclei were stained using 4,6-diamidino-2-phenylindole (DAPI; Invitrogen; Thermo Fisher Scientific, Inc.). Images of cells were captured using fluorescence microscopy (Leica Microsystems).

Reverse-transcription quantitative (RT-q)PCR. To detect the ability for differentiation, several representative genes, INSULIN, glucose transporter 2 (GLUT-2) and neurogenin 3 (NGN-3), were analyzed through RT-qPCR. Total RNA was isolated from both induced cells and control cells using TRIzol reagent (Biyuntian) following the manufacturer's protocol. The RNA concentration and quality were assessed with a NanoDrop One spectrophotometer (Thermo Fisher Scientific, Inc.). Subsequently, the RT reaction was performed using the RT reagent kit with gDNA Eraser (Takara Bio, Inc.) according to the manufacturer's protocol. Real-time PCR was performed with the primers presented in Table I in a StepOne Plus System (Thermo Fisher Scientific, Inc.). According to the manufacturer's protocol for the FastStart Universal SYBR-Green Master (cat. no. 04913914001; Roche Diagnostics), the PCR mixture was prepared and the PCR parameters were set. The thermocycling conditions use were as follows: $95^{\circ} \mathrm{C}$ for $10 \mathrm{~min}$, followed by 40 cycles at $95^{\circ} \mathrm{C}$ for $15 \mathrm{sec}$ and at $60^{\circ} \mathrm{C}$ for $60 \mathrm{sec}$. Melting curve analysis was performed at the end of RT-qPCR. The results were calculated through $2^{-\triangle \Delta C q}$ method and normalized to GAPDH and the control group (27).
Quantitative insulin secretion assay. After differentiation, the induction medium was removed and the cells were washed twice with PBS. New medium with high or low glucose was added and the supernatants were collected after glucose stimulation for $4 \mathrm{~h}$. The concentration of insulin in the supernatants collected from the three types of MSCs in high or low glucose medium was determined using the Human Insulin ELISA kit (cat. no. KAQ1251; Invitrogen; Thermo Fisher Scientific, Inc.). The experiments were performed following the according to the manufacturer's protocol of the ELISA kit. Absorbance was read at $450 \mathrm{~nm}$. The standard curve was constructed using the absorbance of the standard sample.

Statistical analysis. All values were expressed as the mean \pm standard deviation. Comparisons between two groups were analyzed with the unpaired Student's t-test. Comparisons between more than two groups were analyzed with one-way analysis of variance (ANOVA) followed by least-significant differences post-hoc tests for equal variances assumed and Tamhane's T2 post-hoc tests if no equal variances were assumed. A two-way ANOVA with Tukey's post-hoc test was used for comparisons between two factors. For non-parametric data, a Kruskal-Wallis test with Dunn's post-hoc test was used for grading scale analysis. $\mathrm{P}<0.05$ was considered to indicate statistical significance. All analyses were performed with SPSS 23.0 (IBM Corporation).

\section{Results}

Morphology of MENSCs, UCMSCs and DPSCs. Most of the primary MENSCs, UCMSCs and DPSCs adhered to the culture plates within $24 \mathrm{~h}$ after seeding and exhibited a polygonal or round morphology. The cells presented with pseudopodia and displayed similar spindle-shaped morphology for $\sim 2$ days of culture (Fig. 1A).

Expression of cell surface markers on MENSCs, UCMSCs and DPSCs. The expression of the surface receptor molecules CD14, CD29, CD34, CD44, CD45 and CD90 on MENSCs, UCMSCs and DPSCs was determined through flow cytometry. The results indicated that MENSCs, UCMSCs and DPSCs were all positive for CD29, CD44 and CD90 and negative for CD14, CD34 and CD45, which were consistent with the identification criteria for MSCs (28). The flow cytometry results are provided in Fig. S1. Statistical analysis indicated no 
A

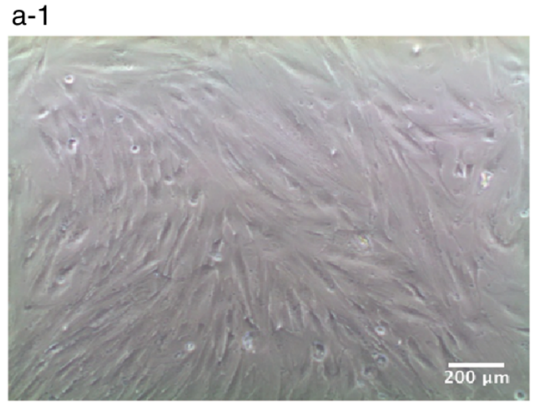

a-2

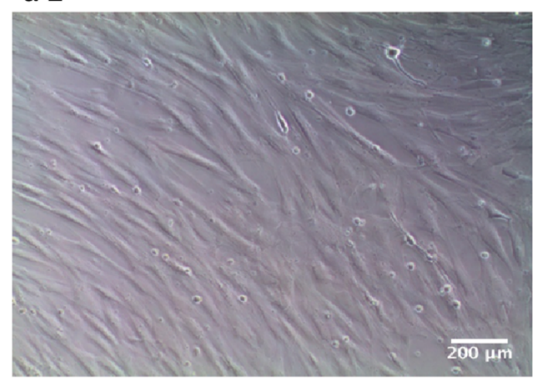

a-3

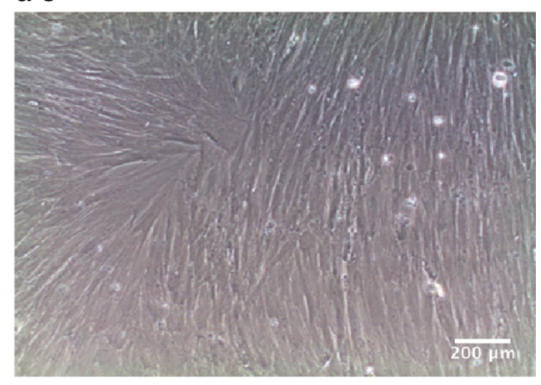

B

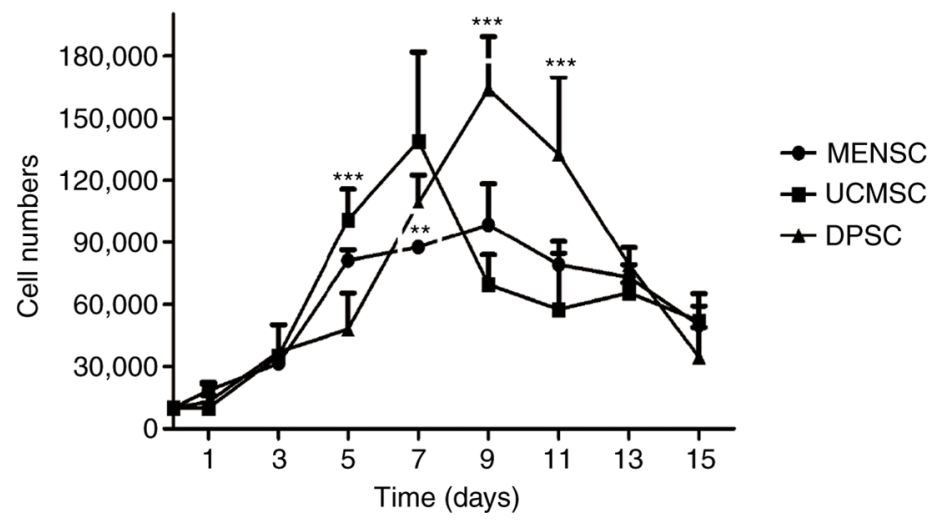

Figure 1. Basic characteristics of the three types of MSC. (A) Morphology of (a-1) P4 MENSCs, (a-2) P4 UCMSCs and (a-3) P4 DPSCs scultured ex vivo (magnification x100; scale bar, $200 \mu \mathrm{m}$ ). (B) Growth curves of MENSCs, UCMSCs and DPSCs. Initial cell seeding: 1.0x10 ${ }^{4}$ cells/cm ${ }^{2}$. Values are expressed as the mean \pm standard deviation of results from three independent experiments. ${ }^{* *} \mathrm{P}<0.01,{ }^{* * * *} \mathrm{P}<0.001$. Significances indicate comparison among three types of MSCs, at the same time point. MENSCs, menstrual blood-derived MSCs; UCMSCs, umbilical cord-derived MSCs; DPSCs, dental pulp MSCs; MSC, mesenchymal stem cell; P4, passage 4; d, days.

significant difference in the surface marker profiles of these three types of cells $(\mathrm{P}>0.05)$.

Growth curves of MENSCs, UCMSCs and DPSCs. Growth curves of MENSCs, UCMSCs and DPSCs were drawn (Fig. 1B) and the PDT was calculated and presented in Table II. The PDT of UCMSCs was the lowest among the three types of MSC, followed by that of DPSCs and then MENSCs. The growth curves also indicated that UCMSCs had the fastest and MENSCs had the slowest proliferation. Specifically, in the first three days, the growth trend of the three types of MSC was similar. At day 7, UCMSCs reached a maximum number of cells, while MENSCs and DPSCs were almost at the maximum at day 9. After 9 days, the number of the three cell types decreased continuously until day 15 , which is related to the contact inhibition induced by the full confluency (29) (Fig. 1B).

Multi-differentiation capabilities of MENSCs, UCMSCs and DPSCs. As indicated in Fig. 2, the three cell types were able to efficiently differentiate into osteocytes and adipocytes. The osteogenesis of UCMSCs was much more prominent and efficient than that of DPSCs and MENSCs. When Alizarin red S staining was performed at the end of week 3, most of the UCMSCs stained red, followed by DPSCs and MENSCs in terms of quantity (Fig. 2A and B). The quantitative results are provided in Fig. 2E. The absorbance of the induced groups of all these MSCs was stronger than that of the control groups, which indicated that all the treated MSCs differentiated into osteoblasts. The absorbance of UCMSCs was stronger than that of MENSCs and DPSCs, which was consistent with the observations in the staining images.

For adipogenic induction, UCMSCs exhibited the best differentiation capability among the three types of MSC. The Oil red $\mathrm{O}$ staining for adipocytes was more prominent for UCMSCs compared with that of MENSCs and DPSCs (Fig. 2C and D). The quantitative results are presented in Fig. 2F. The absorbance of the induced groups of all of these MSCs was stronger than that of the control groups, which indicated that all of these MSCs differentiated into adipocytes. The absorbance of UCMSCs and MENSCs was stronger than that of DPSCs.

Pancreatic $\beta$-like cell differentiation ability of MENSCs, UCMSCs and DPSCs

Quantitative analysis of DTZ staining. After induction, MSCs were subjected to staining with DTZ and then observed under the microscope. Stained sample islet cells were red (Fig. 3A and B), as the induced islet cells contain zinc ions that combine with dithizone, producing a palm red color. MENSCs exhibited the most obvious DTZ staining, while UCMSCs and DPSCs did not stain as well as MENSCs. The quantitative staining percentage of MENSCs was higher than that of UCMSCs and DPSCs (Fig. 3C). The results of the graded analysis suggested that the grade of staining of MENSCs was higher than that of UCMSCs and DPSCs and the results were consistent with those quantified with ImageJ (Fig. 3D). 
A a-1

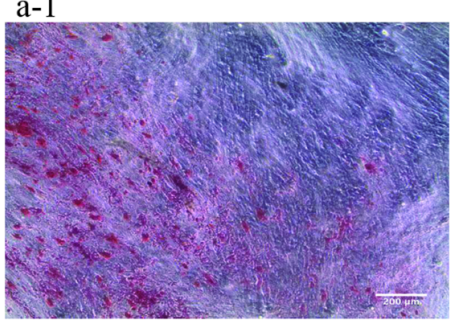

B

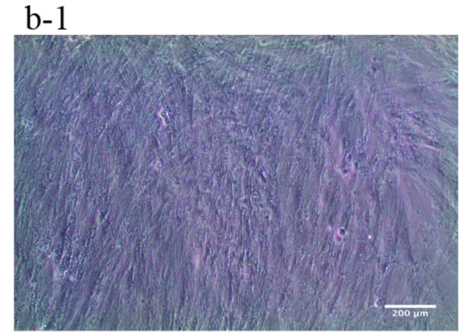

C c-1

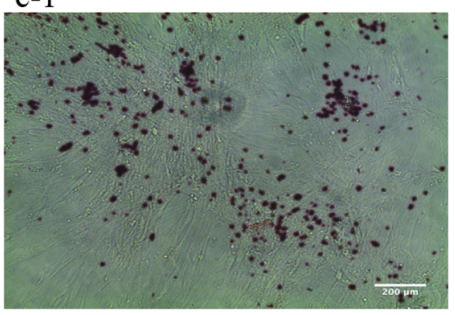

D

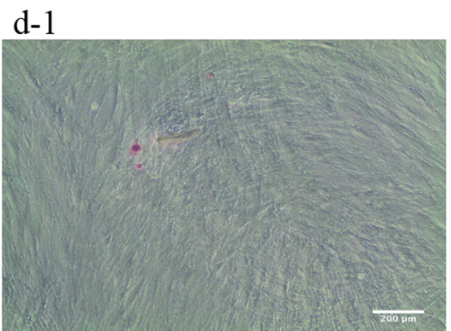

Osteogenic OD-570 nm

E

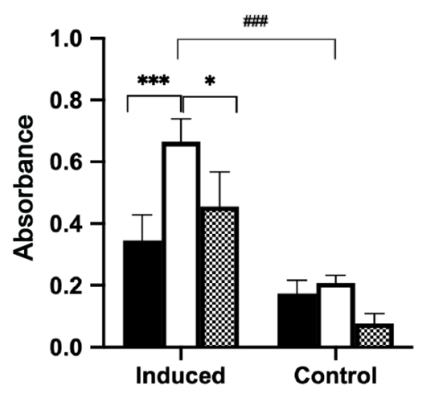

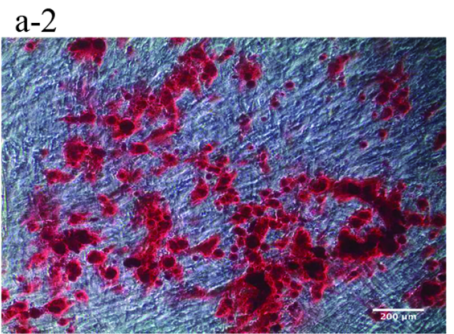

b-2

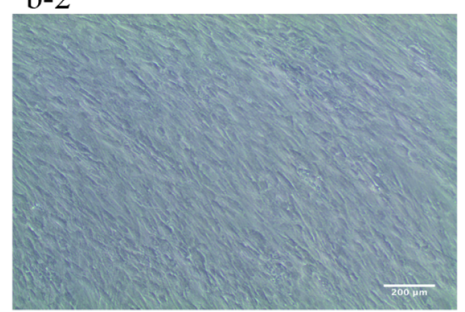

c-2

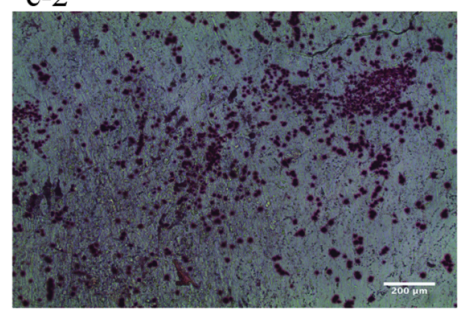

d-2

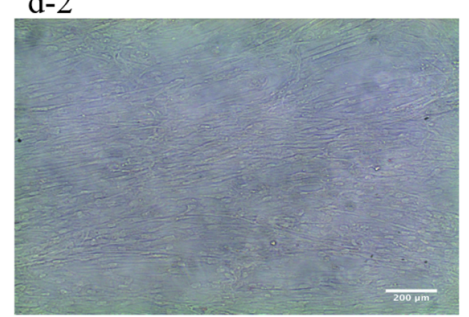

a-3

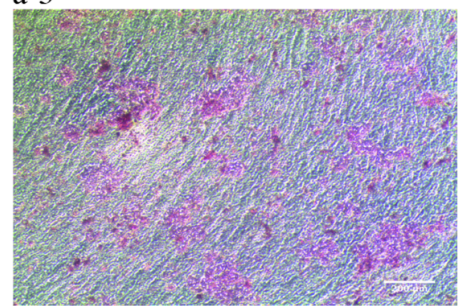

b-3

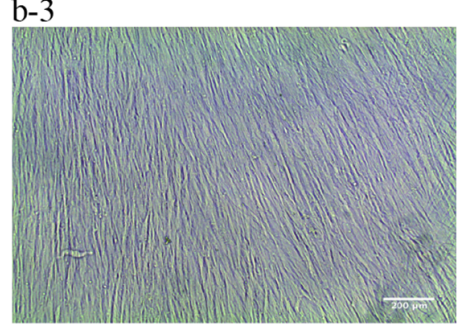

c-3

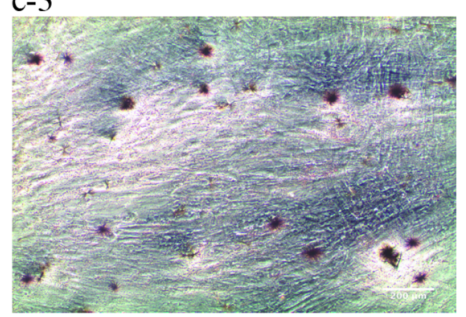

d-3

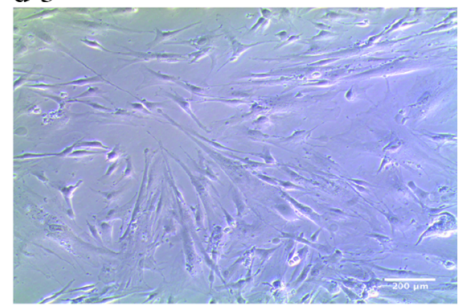

Adipogenic OD-510 nm

$\mathbf{F}$

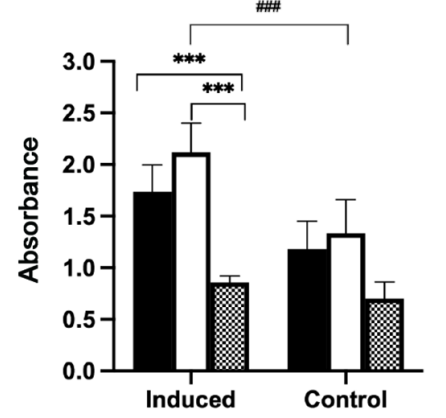

- MENSC

$\square$ UCMSC

$\$$ DPSC $\square$ UCMSC

DPSC

Figure 2. Osteogenic and adipogenic differentiation of the three types of MSC. (A) Osteogenesis differentiation of P4 (a-1) MENSCs, (a-2) UCMSCs and (a-3) DPSCs safter Alizarin red S staining. (B) Control groups for osteogenesis differentiation of P4 (b-1) MENSCs, (b-2) UCMSCs and (b-3) DPSCs after Alizarin red S staining. (C) Adipogenesis differentiation of P4 (c-1) MENSCs, (c-2) UCMSCs and (c-3) DPSCs safter Oil red O staining. (D) Control groups for adipogenesis differentiation of (d-1) MENSCs, (d-2) UCMSCs and (d-3) DPSCs after Oil red O staining (magnification x100; scale bar, $200 \mu$ m). (E) Semiquantitative results of osteogenic differentiation. (F) Semiquantitative results of adipogenic differentiation. ${ }^{*} \mathrm{P}<0.05,{ }^{* * * *} \mathrm{P}<0.001 ;{ }^{\# \# t} \mathrm{P}<0.001$ vs. control. MENSCs, menstrual blood-derived MSCs; UCMSCs, umbilical cord-derived MSCs; DPSCs, dental pulp MSCs; MSC, mesenchymal stem cell; OD, optical density; P4, passage 4.

Insulin expression by laser immune confocal microscopy. After the three-step induction, the MENSCs tested positive for insulin expression, while the UCMSCs were negative for insulin and the DPSCs exhibited weak expression (Fig. 4). DAPI-stained nuclei appeared blue and cells that expressed insulin appeared red. It was proved that the induced MENSCs obtained a synthetic insulin function. The ability of MENSCs to produce insulin was the best, followed by that of DPSCs and then that of UCMSCs. 


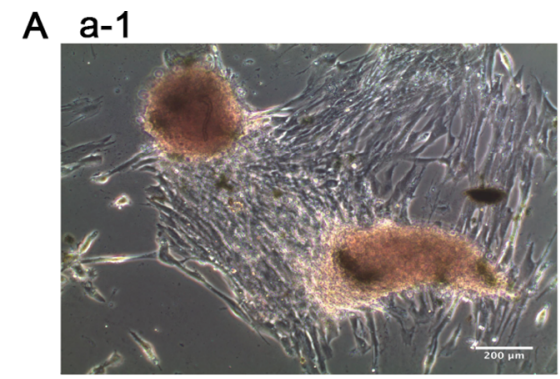

B

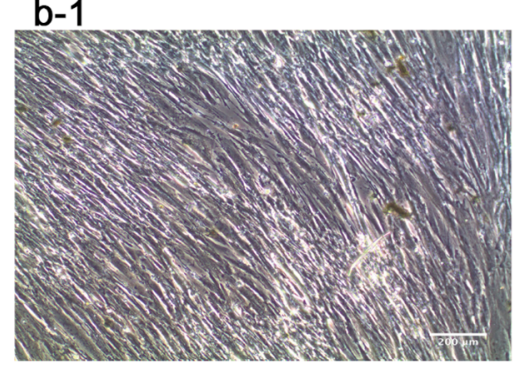

a-2

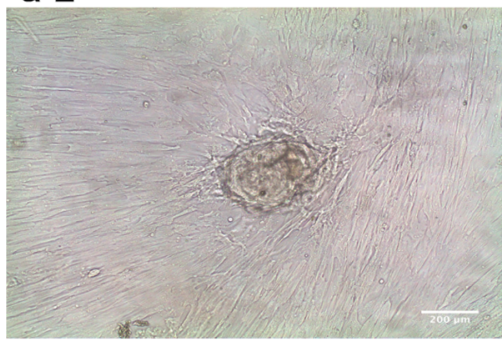

b-2

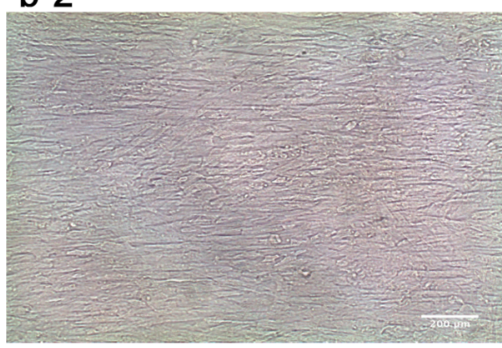

a-3

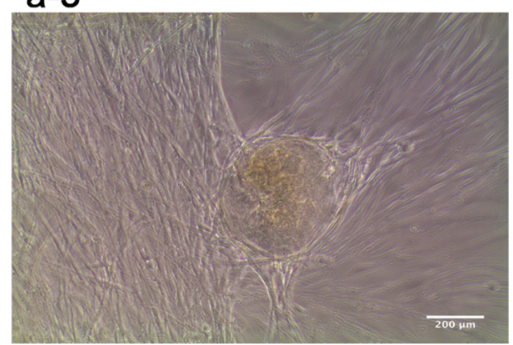

b-3

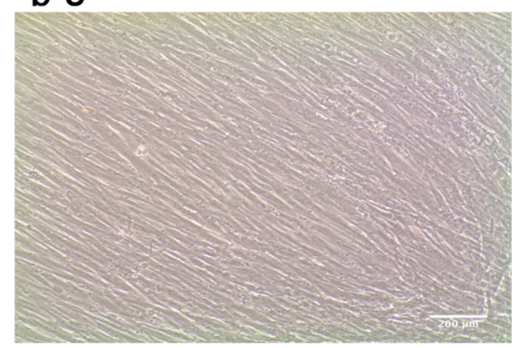

C

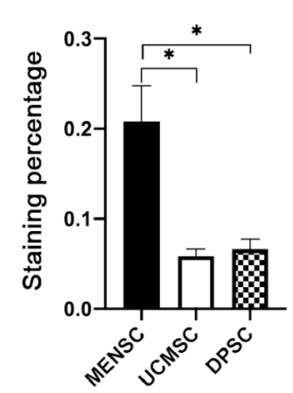

D

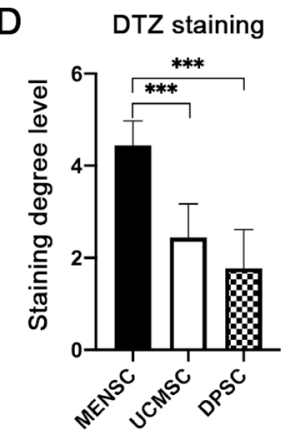

Figure 3. Results of differentiation into pancreatic $\beta$-like cells. (A) DTZ staining of P4 (a-1) MENSCs, (a-2) UCMSCs and (a-3) DPSCs. (B) The DTZ staining results of undifferentiated groups of P4 (b-1) MENSCs, (b-2) UCMSCs and (b-3) DPSCs (magnification x100; scale bar, $200 \mu \mathrm{m}$ ). (C) Quantitative results of the DTZ staining. The percentage of the whole microscopic imageswas determined with ImageJ software, imitating immunohistochemistry. (D) Quantitative results of DTZ staining with grading from $1-5$. ${ }^{*} \mathrm{P}<0.05,{ }^{* * * *} \mathrm{P}<0.001$. MENSCs, menstrual blood-derived MSCs; UCMSCs, umbilical cord-derived MSCs; DPSCs, dental pulp MSCs; MSC, mesenchymal stem cell; DTZ, dithizone; P4, passage 4.

Expression of genes characteristic for islet cells. The expression of islet-related genes was analyzed by using $R T$ - $q P C R$ (Fig. 5). The iconic genes INSULIN, GLUT-2 and NGN-3 were detected. Subsequent to differentiation, the expression of INSULIN and GLUT-2 in MENSCs was significantly higher than that in the control group. The expression of NGN3 was slightly higher in the induced group with no significance. Furthermore, the expression of INSULIN and GLUT-2 in UCMSCs after induction was lower than that in the control group. The expression of NGN3 was not altered after induction. The amount of NGN-3 expressed in the DPSCs was much higher than that in the control group $(\mathrm{P}<0.01)$. The average INSULIN expression of the induced group was slightly higher than that of the control group, while GLUT-2 expression of the induced group was lower.

Insulin secretion by ELISA. The insulin secretion for the three types of adult MSCs varied according to the different glucose concentrations in the medium (Fig. 6). The level of insulin secreted by MENSCs induced in vitro into islet cells exposed to high glucose concentration was $11.95 \pm 0.61 \mathrm{mU} / 1$. For UCMSCs induced in the presence of high glucose, the level of secreted insulin was $6.61 \pm 0.37 \mathrm{mU} / 1$ and that of DPSCs was $5.26 \pm 0.66 \mathrm{mU} / \mathrm{l}$. When the glucose concentration was $5.5 \mathrm{mmol} / \mathrm{l}$, the insulin secretion of induced MENSCs was $3.46 \pm 0.29 \mathrm{mU} / \mathrm{l}$, while that of UCMSCs was $2.75 \pm 0.39 \mathrm{mU} / \mathrm{l}$ and that of DPSCs was $4.11 \pm 0.42 \mathrm{mU} / 1$. Additional insulin was not secreted after low glucose stimulation both in induced and control MSCs, which indicated that the secretion of insulin was dependent on glucose stimulation in the medium. According to the results, it was observed that induced MSCs secreted higher concentration of extracellular insulin compared with control MSCs when exposed to high glucose concentration of $25 \mathrm{mmol} / \mathrm{l}$. When induced MSCs were exposed to high glucose concentration, MENSCs secreted the highest amount of insulin, followed by UCMSCs and then DPSCs. Based on these results, MENSCs were more suitable than UCMSCs and DPSCs as seed cell candidates for the induction of islet cells in vitro.

\section{Discussion}

In the present study, the ability of three types of MSC to differentiate into pancreatic $\beta$-like cells in vitro was compared. Other studies reported the use of diverse methods to differentiate MSCs toward a pancreatic cell lineage (30-33). A three-step method to effectively induce MENSCs, UCMSCs and DPSCs to differentiate into pancreatic $\beta$-like cells 
A

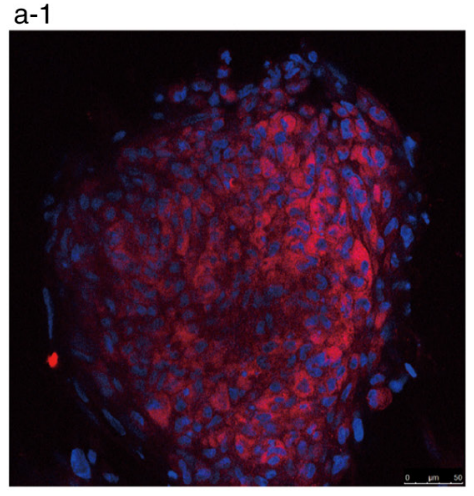

a-2

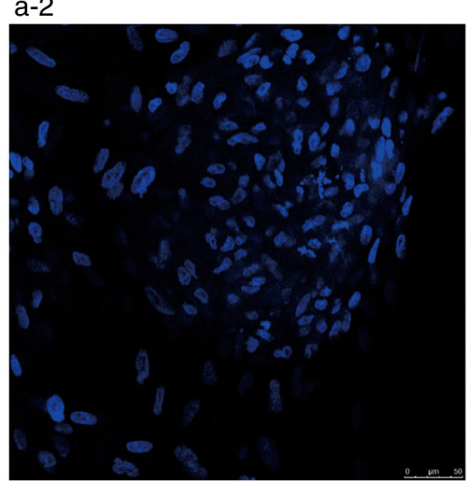

B

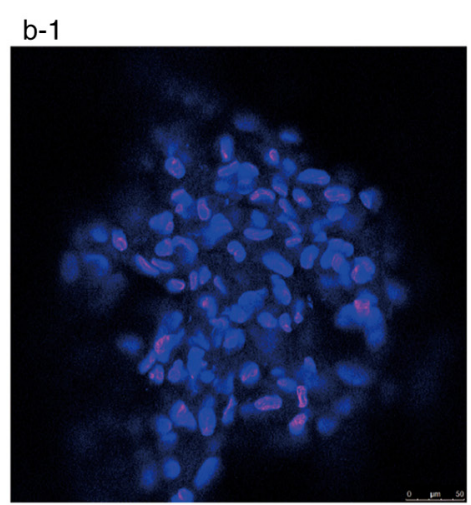

b-2

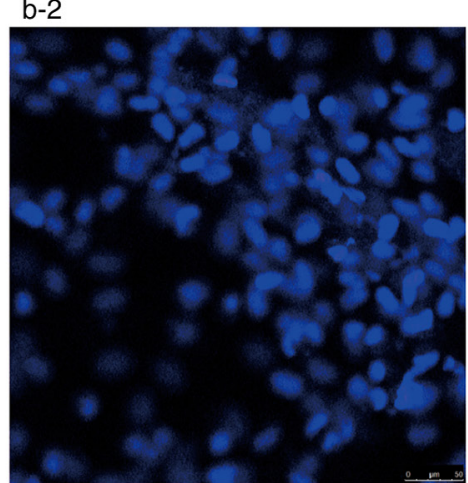

C

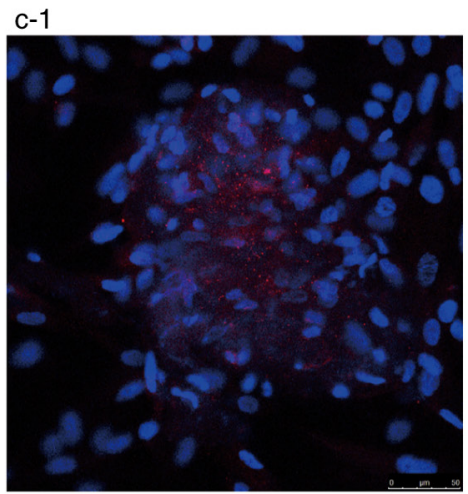

$c-2$

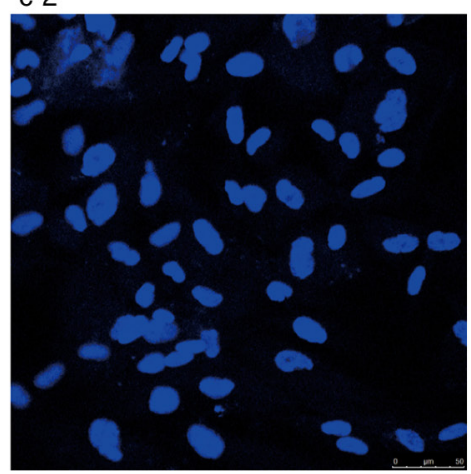

Figure 4. Confocal microscopy imagesof the three types of MSC at passage 4 insulin protein expressed in MSCs stained with anti-insulin primary antibody and tetramethylrhodamine-conjugated secondary antibody. Cell nuclei were stained using DAPI. (A) MENSCs: (a-1) Induced and (a-2) control. (B) UCMSCs: (b-1) Induced and (b-2) control. (C) DPSCs: (c-1) Induced and (c-2) control (magnification x630; scale bar, $50 \mu \mathrm{m}$ ). MENSCs, menstrual blood-derived MSCs; UCMSCs, umbilical cord-derived MSCs; DPSCs, dental pulp MSCs; MSC, mesenchymal stem cell.

A

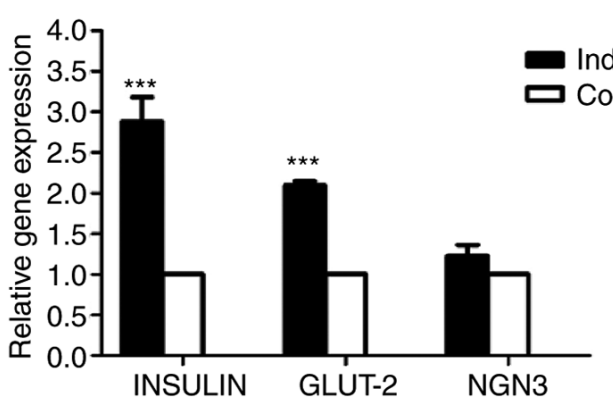

$\mathrm{B}$

Induced group Control group

$\mathrm{C}$

DPSC
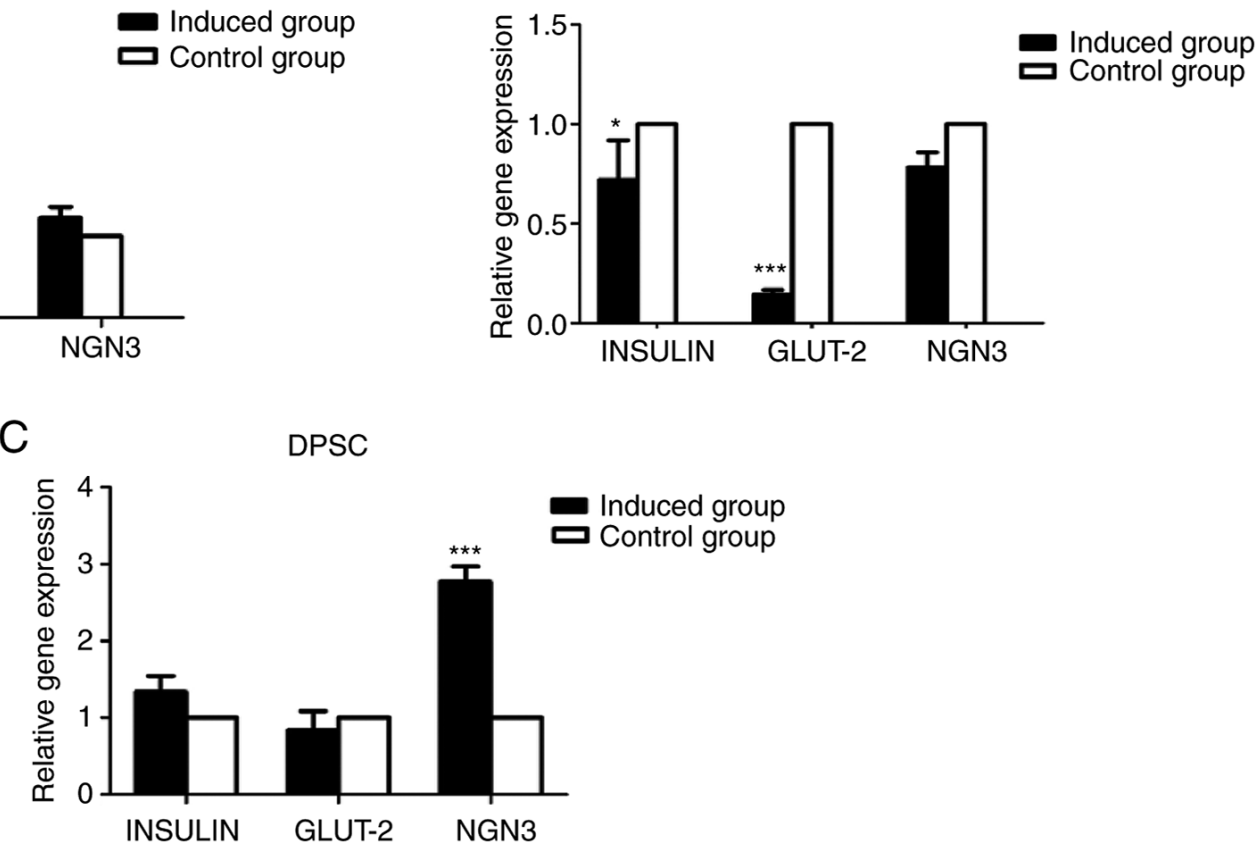

Figure 5. Relative quantification of pancreatic $\beta$-cell gene expression in three MSCs. (A) MENSCs, (B) UCMSCs and (C) DPSCs. The gene expression in the induced group was compared with that in the corresponding control group. The data of the control group and induced group were divided by the control group. The control group was set as 1 and the induced group was expressed as a percentage of the control group. The mRNA levels were normalized using the expression of the reference gene $(\mathrm{GAPDH})$. Values are expressed as the mean \pm standard deviation of results from three independent experiments. ${ }^{*} \mathrm{P}<0.05$, ${ }^{* * * *} \mathrm{P}<0.001$. MENSCs, menstrual blood-derived MSCs; UCMSCs, umbilical cord-derived MSCs; DPSCs, dental pulp MSCs; MSC, mesenchymal stem cell; GLUT-2, glucose transporter 2; NGN-3, neurogenin 3. 


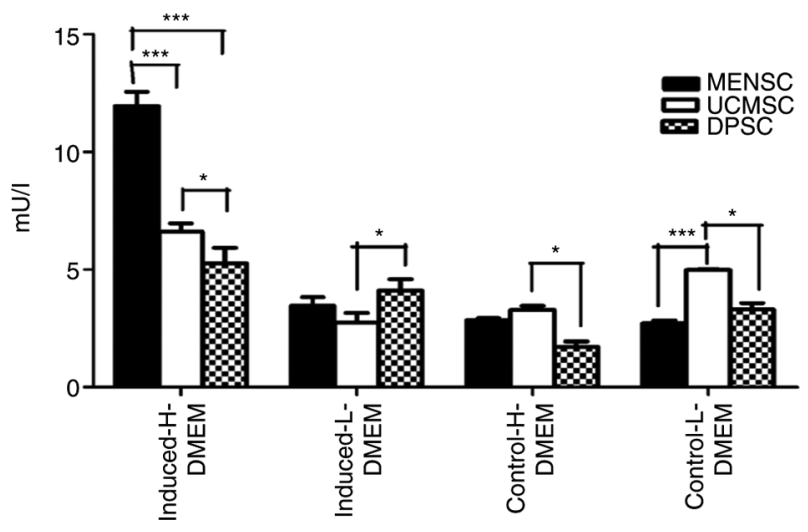

Figure 6. ELISA test results of the three types of MSC. The insulin concentration of induced and control MSCs was measured after exposure to H-DMEM and L-DMEM. ${ }^{*} \mathrm{P}<0.05,{ }^{* * * *} \mathrm{P}<0.001$. MENSCs, menstrual blood-derived MSCs; UCMSCs, umbilical cord-derived MSCs; DPSCs, dental pulp MSCs; MSC, mesenchymal stem cell; H-DMEM, high glucose DMEM; L-DMEM, low glucose DMEM.

in vitro was developed. As described by Veres et al (34), the stem cell-derived $\beta$ (SC- $\beta$ ) cell protocol led to stem cell differentiation into six stages. These six stages were divided into two major steps during differentiation (35), formation of pancreatic progenitors and maturation of insulin-expressing SC- $\beta$ cells. Based on this flow, the protocols were improved according to the preliminary conditions tested in the present study results of our previous study. It was observed that all of the different MSC types were similar in cell surface markers and basic biological characteristics. Following the application of the three-step differentiation method, MENSCs had a strong ability to form spherical shapes. The formation of spherical cells in UCMSCs and DPSCs was less than that of MENSCs. Differences were not only observed in terms of morphology, but also in the staining behavior. DTZ staining was evident in MENSCs, whereas UCMSCs and DPSCs were evidently not stained. In the present study, the expression of insulin was also analyzed through confocal laser microscopy and the results indicated that the production of insulin by MENSCs was much higher than that in DPSCs and UCMSCs. As specific genes of pancreatic $\beta$-cells, INSULIN, GLUT- 2 and NGN-3 were then analyzed. The expression of the three genes in MENSCs was positive compared to that in the undifferentiated control group. According to the results, MENSCs had a stronger ability to differentiate into pancreatic cells than DPSCs and UCMSCs, which means that MENSCs are a novel suitable source for the curative treatment of TID. It was preliminarily concluded that MENSCs have potential for the treatment of TID. However, further studies, such as those using flow cytometry and additional gene expression analyses, are required to confirm this conclusion. Flow cytometry may be applied to quantitatively study the efficiency of differentiation and the percentage of insulin-positive cells after induction (34). Flow cytometry is also beneficial to the qualitative analysis of the differentiation potency of the three types of MSC. NGN-3, GLUT-2, pancreatic and duodenal homeobox 1 (PDX-1), INSULIN, glucagon and somatostatin are iconic genes for the differentiation of pancreatic cells (36). The present study explored the changes in the expression of NGN-3,
INSULIN and GLUT2. The expression of PDX-1, glucagon and somatostatin remain to be explored using RT-qPCR or confocal laser microscopy to strengthen the results of the present study.

Previous studies have demonstrated the differentiation ability of MENSCs, UCMSCs and DPSCs $(9,37,38)$. To the best of our knowledge, the present study was the first to compare the ability of the three stem cell types to differentiate into pancreatic $\beta$-like cells using the same differentiation protocol in the same article.

In the present study, the use of collagen coating enhanced the ability of MSCs to form pancreatic $\beta$-like cells. It was previously reported that the collagen/hyaluronic acid scaffold was able to enhance the differentiation ability of ADSCs into insulin-producing cells (39). Sefcik et al (17) utilized collagen to enhance the osteogenic differentiation ability of ADSCs in vitro.

In the present study, it was speculated that the different differentiation abilities of the MSCs were due to the physiological location of these cells. Suman et al (40) concluded that MSCs are types of germ layer specific multipotent stem cells (MPSCs), which can give rise to monopotent stem cells, and monopotent stem cells differentiate into one adult cell lineage. The pancreas split belongs to the endoderm (41), and endodermal MPSCs tend to differentiate into monopotent stem cells for pancreas, lung and liver cell lineages. The location of the endometrium where the MENSCs are collected is close to endoderm in physiological characteristics (42). It was reported that UCMSCs may express a low amount of ectodermal and endodermal markers, and the majority of the cells express mesodermal markers after cultivation for several days (43). The dental pulp begins in the molar pulp and is derived from the ectoderm originated from migrating neural crest cells (44). According to previous studies, the formation of the definitive endoderm (DE) lineage was essential but not necessary for the differentiation of pancreatic cells, which have the ability to produce insulin and react to high glucose concentrations (45). Based on the results, the MENSCs from the endoderm had a stronger ability to form pancreatic $\beta$-like cells than UCMSCs and DPSCs without the formation of the DE lineage, which indicated that MENSCs are a more suitable source for TID treatment. However, it remains elusive whether the location germ layers where MSCs are derived from serve a role in their differentiation potency into pancreatic cells and this remains to be explored.

In conclusion, in the present study, the differentiation capability of MENSCs, UCMSCs and DPSCs into pancreatic $\beta$-like cells was compared. The MENSCs had the best differentiation ability into pancreatic beta-like cells than UCMSCs and DPSCs. The results demonstrated that the use of MENSCs as a potential new source of progenitor cells for application in the treatment of diabetes was more effective than the use of UCMSCs and DPSCs in vitro.

\section{Acknowledgements}

Not applicable.

\section{Funding}

This project was supported by the National Natural Science Foundation of China (grant. no. 81871122). 


\section{Availability of data and materials}

The datasets used and/or analyzed during the current study are available from the corresponding author on reasonable request.

\section{Authors' contributions}

YTC conceived the present study and supervised it. YFM, ZJW and YTC designed and initiated the experiments. YFM, ZJW, JG and YY performed the experiments. YFM, ZJW, JG, YY, FLZ, HJR and NMQ analyzed the data. YFM and FLZ revised the manuscript. ZJW, NMQ and YTC confirmed the authenticity of the raw data. All authors provided input for the paper writing and discussion during the whole period. All authors have read and approved the final manuscript.

\section{Ethics approval and consent to participate}

Written informed consent was obtained from each donor and the present study was approved by the Ethics Committee at the School of Pharmacy, Shanghai Jiaotong University (Shanghai, China).

\section{Patient consent for publication}

Not applicable.

\section{Competing interests}

The authors declare that they have no competing interests.

\section{References}

1. Stumvoll M, Goldstein BJ and van Haeften TW: Type 2 diabetes: Principles of pathogenesis and therapy. Lancet 365: 1333-1346, 2005.

2. Senior PA and Pettus JH: Stem cell therapies for type 1 diabetes: Current status and proposed road map to guide successful clinical trials. Diabet Med 36: 297-307, 2019.

3. Pixley JS: Mesenchymal stem cells to treat type 1 diabetes. Biochim Biophys Acta Mol Basis Dis 1866: 165315, 2020.

4. Carlsson PO, Schwarcz E, Korsgren O and Le Blanc K: Preserved $\beta$-cell function in type 1 diabetes by mesenchymal stromal cells. Diabetes 64: 587-592, 2015.

5. Troyer DL and Weiss ML: Concise review: Wharton's jelly-derived cells are a primitive stromal cell population. Stem Cells 26: 591-599, 2008.

6. Novais A, Lesieur J, Sadoine J, Sliman L, Baroukh B, Saubaméa B, Schmitt A, Vital S, Poliard A, Hélary C, et al Priming dental pulp stem cells from human exfoliated deciduous teeth with fibroblast growth factor-2 enhances mineralization within tissue-engineered constructs implanted in craniofacial bone defects. Stem Cells Transl Med 8: 844-857, 2019.

7. Liu Y, Niu R, Li W, Lin J, Stamm C, Steinhoff G and Ma N: Therapeutic potential of menstrual blood-derived endometrial stem cells in cardiac diseases. Cell Mol Life Sci 76: 1681-1695, 2019.

8. Asahara T, Kalka C and Isner JM: Stem cell therapy and gene transfer for regeneration. Gene Ther 7: 451-457, 2000

9. Brown C, McKee C, Bakshi S, Walker K, Hakman E, Halassy S, Svinarich D, Dodds R, Govind CK and Chaudhry GR: Mesenchymal stem cells: Cell therapy and regeneration potential. J Tissue Eng Regen Med 13: 1738-1755, 2019.

10. Shivakumar SB, Bharti D, Subbarao RB, Park JM, Son YB, MUllah I, Choe YH, Lee HJ, Park BW, Lee SL and Rho GJ: Pancreatic endocrine-like cells differentiated from human umbilical cords wharton's jelly mesenchymal stem cells using small molecules. J Cell Physiol 234: 3933-3947, 2019.
11. Pedroni ACF, Sarra G, de Oliveira NK, Moreira MS, Deboni MCZ and Marques MM: Cell sheets of human dental pulp Stem cells for future application in bone replacement. Clin Oral Investig 23: 2713-2721, 2019

12. Liu JJ, Yu F, Sun Y, Jiang B, Zhang W, Yang J, Xu GT, Liang A and Liu S: Concise reviews: Characteristics and potential applications of human dental tissue-derived mesenchymal stem cells. Stem Cells 33: 627-638, 2015.

13. Patel AN, Park E, Kuzman M, Benetti F, Silva FJ and Allickson JG: Multipotent menstrual blood stromal stem cells: Isolation, characterization, and differentiation. Cell Transplant 17: 303-311, 2008.

14. Shivakumar SB, Lee HJ, Son YB, Bharti D, Ock SA, Lee SL, Kang YH, Park BW and Rho GJ: In vitro differentiation of single donor derived human dental mesenchymal stem cells into pancreatic beta cell-like cells. Biosci Rep 39: BSR20182051, 2019.

15. Linh NTB, Abueva CDG, Jang DW and Lee BT: Collagen and bone morphogenetic protein-2 functionalized hydroxyapatite scaffolds induce osteogenic differentiation in human adipose-derived stem cells. J Biomed Mater Res Part B Appl Biomater 108: 1363-1371, 2019.

16. Bilic-Curcic I, Kalajzic Z, Wang L and Rowe DW: Origins of endothelial and osteogenic cells in the subcutaneous collagen gel implant. Bone 37: 678-687, 2005.

17. Sefcik LS, Neal RA, Kaszuba SN, Parker AM, Katz AJ, Ogle RC and Botchwey EA: Collagen nanofibres are a biomimetic substrate for the serum-free osteogenic differentiation of human adipose stem cells. J Tissue Eng Regen Med 2: 210-220, 2008.

18. Ren H, Sang Y, Zhang F, Liu Z, Qi N and Chen Y: Comparative analysis of human mesenchymal stem cells from umbilical cord, dental pulp, and menstrual blood as sources for cell therapy. Stem Cells Int 2016: 3516574, 2016.

19. Skyler JS, Fonseca VA, Segal KR, Rosenstock J; MSB-DM003 Investigators: Allogeneic mesenchymal precursor cells in type 2 diabetes: A randomized, placebo-controlled, dose-escalation safety and tolerability pilot study. Diabetes Care 38: 1742-1749, 2015.

20. Trivedi HL, Thakkar UG, Vanikar AV and Dave SD: Treatment of polyglandular autoimmune syndrome type 3 using co-transplantation of insulin-secreting mesenchymal stem cells and haematopoietic stem cells. BMJ Case Rep 2011: bcr0720114436, 2011.

21. Liu X, Zheng P, Wang X, Dai G, Cheng H, Zhang Z, Hua R, Niu X, Shi J and An Y: A preliminary evaluation of efficacy and safety of Wharton's jelly mesenchymal stem cell transplantation in patients with type 2 diabetes mellitus. Stem Cell Res Ther 5: 57, 2014.

22. Hoveizi E and Mohammadi T: Differentiation of endometrial stem cells into insulin-producing cells using signaling molecules and zinc oxide nanoparticles, and three-dimensional culture on nanofibrous scaffolds. J Mater Sci Mater Med 30: 101, 2019.

23. Santamaria X, Massasa EE, Feng YZ, Wolff E and Taylor HS: Derivation of insulin producing cells from human endometrial stromal stem cells and use in the treatment of murine diabetes. Mol Ther 19: 2065-2071, 2011.

24. Shiroi A, Yoshikawa M, Yokota H, Fukui H, Ishizaka S, Tatsumi K and Takahashi Y: Identification of insulin-producing cells derived from embryonic stem cells by zinc-chelating dithizone. Stem Cells 20: 284-292, 2002.

25. Shu J, Dolman GE, Duan J, Qiu GP and Ilyas M: Statistical colour models: An automated digital image analysis method for quantification of histological biomarkers. Biomed Eng Online 15: 46, 2016.

26. Gibson-Corley KN, Olivier AK and Meyerholz DK: Principles for valid histopathologic scoring in research. Vet Pathol 50: 1007-1015, 2013.

27. Livak KJ and Schmittgen TD: Analysis of relative gene expression data using real-time quantitative PCR and the 2(-Delta Delta C(T)) method. Methods 25: 402-408, 2001.

28. Dominici M,Le Blanc K, Mueller I, Slaper-Cortenbach I, Marini F, Krause D, Deans R, Keating A,Prockop D and Horwitz E: Minimal criteria for defining multipotent mesenchymal stromal cells. The international society for cellular therapy position statement. Cytotherapy 8: 315-317, 2006.

29. Zhou YF, Bosch-Marce M, Okuyama H, Krishnamachary B, Kimura H, Zhang L, Huso DL and Semenza GL: Spontaneous transformation of cultured mouse bone marrow-derived stromal cells. Cancer Res 66: 10849-10854, 2006.

30. Van Pham P, Nguyen PTM, Nguyen ATQ, Pham VM, Bui ANT, Dang LTT, Nguyen KG and Phan N: Improved differentiation of umbilical cord blood-derived mesenchymal stem cells into insulin-producing cells by PDX-1 mRNA transfection. Differentiation 87: 200-208, 2014. 
31. Duruksu G and Aciksari A: Guiding the differentiation direction of pancreatic islet-derived stem cells by glycated collagen. Stem Cells Int 3: 6143081, 2018.

32. Gao F, Wu Y, Wen H, Zhu W, Ren H, Guan W and Tian X: Multilineage potential research on pancreatic mesenchymal stem cells of bovine. Tissue Cell 56: 60-70, 2019.

33. Phadnis SM, Joglekar MV, Dalvi MP, Muthyala S, Nair PD, Ghaskadbi SM, Bhonde RR and Hardikar AA: Human bone marrow-derived mesenchymal cells differentiate and mature into endocrine pancreatic lineage in vivo. Cytotherapy 13: 279-293, 2011.

34. Veres A, Faust AL, Bushnell HL, Engquist EN, Kenty JHR, Harb G, Poh YC, Sintov E, Gürtler M, Pagliuca FW, et al: Charting cellular identity during human in vitro beta-cell differentiation. Nature 569: 368-373, 2019.

35. Manaph NPA, Sivanathan KN, Nitschke J, Zhou XF, Coates PT and Drogemuller CJ: An overview on small molecule-induced differentiation of mesenchymal stem cells into beta cells for diabetic therapy. Stem Cell Res Ther 10: 18, 2019.

36. Chandra V, Swetha G, Phadnis S, Nair PD and Bhonde RR: Generation of pancreatic hormone-expressing islet-like cell aggregates from murine adipose tissue-derived stem cells. Stem Cells 27: 1941-1953, 2009.

37. Chen LJ, Qu JJ and Xiang C: The multi-functional roles of menstrual blood-derived stem cells in regenerative medicine. Stem Cell Res Ther 10: 10, 2019.

38. Xing YX, Zhang YP, Wu X, Zhao B, Ji YW and Xu X: A comprehensive study on donor-matched comparisons of three types of mesenchymal stem cells-containing cells from human dental tissue. J Periodont Res 54: 286-299, 2019.
39. Khorsandi L, Khodadadi A, Nejad-Dehbashi F and Saremy S Three-dimensional differentiation of adipose-derived mesenchymal stem cells into insulin-producing cells. Cell Tissue Res 361: 745-753, 2015.

40. Suman S, Domingues A, Ratajczak J and Ratajczak MZ: Potential clinical applications of stem cells in regenerative medicine. Adv Exp Med Biol 1201: 1-22, 2019.

41. Bastidas-Ponce A, Scheibner K, Lickert H and Bakhti M: Cellular and molecular mechanisms coordinating pancreas development. Development 144: 2873-2888, 2017.

42. Lin J, Xiang D, Zhang JL, Allickson J and Xiang C: Plasticity of human menstrual blood stem cells derived from the endometrium. J Zhejiang Univ Sci B 12: 372-380, 2011.

43. Bahmanpour S, Khozani TT and Tazangi FR: Evaluation of the capability of the Wharton's jelly mesenchymal stem cell aggregates to express the markers of three germ cell lineages. Arch Iran Med 22: 85-90, 2019.

44. Lan X, Sun Z, Chu C, Boltze J and Li S: Dental pulp stem cells: An attractive alternative for cell therapy in ischemic stroke. Front Neurol 10: 824, 2019.

45. Baetge EE: Production of beta-cells from human embryonic stem cells. Diabetes Obes Metab 10: 186-194, 2008.

(i) (3) This work is licensed under a Creative Commons Attribution-NonCommercial-NoDerivatives 4.0 International (CC BY-NC-ND 4.0) License. 